\title{
Universal health coverage for strengthening prevention and control of noncommunicable diseases in COVID-19 era
}

\author{
Amirhossein Takian*1,2,3 (1), Ahad Bakhtiari ${ }^{2,4}$, Afshin Ostovar ${ }^{5}$ \\ Received: 8 Aug 2020 \\ Published: 11 Nov 2020
}

\section{Abstract}

The novel coronavirus disease (COVID-19) pandemic has been exhausting the entire global economy. As the greatest challenge to sustainable development in all societies and health systems, noncommunicable diseases (NCDs) and their relevant risk factors are the main causes of illness and death during the 21 st century in high, middle, and low-income countries (LMICs). NCDs are also among the main underlying causes of death among COVID-19 patients in many countries. People living with or affected by NCDs (PLWANCDs) are more vulnerable to becoming severely ill with COVID-19. Although the ongoing pandemic will be a fundamental game-changer for prioritization and resource allocation in many countries in years to come, ample evidence indicates that NCDs will remain the main killer of people and the costliest barrier to sustainable societies. Looking through the lenses of universal health coverage (UHC), this paper advocates rebuilding our world during COVID-19 aftermath, in a way to harmonize efforts to live with pandemics and make our health systems resilient, balanced, and comprehensive enough to accommodate all threats to humanity, including both communicable and noncommuincable diseases.

Keywords: COVID-19, Pandemic, Noncommunicable disease (NCD), Universal health coverage (UHC)

Conflicts of Interest: AT and AO are members of the INCDC at the MoHME-Iran. AO is Director-General for NCDs at the MoHME. We have no conflict of interest to disclose. Funding: None

*This work has been published under CC BY-NC-SA 1.0 license.

Copyright $\odot$ Iran University of Medical Sciences

Cite this article as: Takian A, Bakhtiari A, Ostovar A. Universal health coverage for strengthening prevention and control of noncommunicable diseases in COVID-19 era. Med J Islam Repub Iran. 2020 (11 Nov);34:153. https://doi.org/10.47176/mjiri.34.153

\section{Introduction}

Since its first appearance on December 30, 2019 in China , and following the pandemic of the novel coronavirus disease (COVID-19) on March 11, 2020, the capacity and resilience of health systems and the entire economy of countries from across the globe have been exhausting to combat the crisis (1). As of May 29, 2020, over 5.8 million people have been identified, while 360000 died and over 2.5 million recovered in 214 countries and territories (2). Described as the greatest challenge of all countries after the

Corresponding author:Dr Amirhossein Takian, takian@tums.ac.ir

1. Department of Global Health and Public Policy, School of Public Health, Tehran University of Medical Sciences, Tehran, Iran

2. Department of Health Management and Economics, School of Public Health, Tehran University of Medical Sciences, Tehran, Iran

3. Health Equity Research Centre (HERC), Tehran University of Medical Sciences, Tehran, Iran

4. National Center for Health Insurance Research, Iran Health Insurance Organization, Tehran, Iran

5. Osteoporosis Research Center, Endocrinology and Metabolism Clinical Sciences Institute, Tehran University of Medical Sciences, Tehran, Iran
World WarII, COVID-19 and its devastating consequences have brought many countries on their knees by shrinking the global economy (3).

Noncommunicable diseases (NCDs) might be themselves the main underlying cause of death among people dying from COVID-19 in many countries (4). Evidence shows that people living with or affected by NCDs (PLWANCDs) are more vulnerable to becoming severely ill with COVID-19 (5). Preexisting comorbid conditions

\section{$\uparrow$ What is "already known" in this topic:}

COVID-19 is exhausting the health systems in most countries. This ongoing pandemic will be a fundamental game-changer for prioritization and resource allocation in many settings in years to come.

\section{$\rightarrow$ What this article adds:}

NCDs will remain the main killer of people and the costliest barrier to sustainable societies during the 21 st century. We must act now, more than ever, to rebuild our world in a way to make our health system resilient enough to accommodate all threats to humanity, including both communicable and noncommuincable diseases 
are associated with increasing case-fatality rate (CFR) among COVID-19 patients: $10.5 \%$ for cardiovascular disease, $7.3 \%$ for diabetes, $6.3 \%$ for chronic respiratory disease, $6.0 \%$ for hypertension and $5.6 \%$ for cancer with overall CFR 2.3\% (5).

\section{Global efforts for prevention and control of NCDs before COVID-19}

NCDs and their relevant risk factors are the main causes of illness and death during the $21^{\text {st }}$ century in high, lowand-middle income countries (LMICs), with a considerably higher burden in the LMICs. Also, 62\% of total DALYs ( 2.5 billion) and $73 \%$ of total deaths ( 56 million) in 2017 are NCD-related worldwide (6). During the last decade prior to COVID-19 pandemic, the World Health Organization (WHO) has been making its utmost effort to direct the attention of member states to invest more on NCDs and scale up their political leadership and technical capacity to reduce the burden of NCDs by 30\% through 2030 sustainable development goals (SDG) agenda (7).

The global community identified NCDs as the greatest challenge to sustainable development in all societies and health systems (7). The UN Political Declaration of 2011, the global action plan for prevention and control of NCDs of 2013, the UN High-Level meetings of 2014 and 2018, and the Global Coordination Mechanism (GCM), and the UN Interagency Task Force (UNITAF), among other global initiatives, were all put in place with great enthusiasm, ambition and effort to both galvanize the WHO and health systems in many countries with skills, insight, and experience to improve NCDs care, as well as to foster the multisectoral and whole-governance/whole-society approach, which are fundamental measures for prevention and control of NCDs anywhere. By the so-called BestBuys, WHO created an evidence-based and cost-effective list of interventions for health systems to choose from in tackling NCDs and spent great resources to move member states to prioritize NCDs and invest in policies and interventions to beat NCDs.

The implementation of these initiatives saved millions of lives and improved their quality of life. As COVID-19 and its consequences will be absorbing much of health system resources in the near future, we advocate the global public health community to continue the utmost support for prevention and control of NCDs to avoid significant deaths and increase DALYs during COVID-19 aftermath.

\section{Specific response to NCDs during COVID-19 crisis in Iran}

Similar to many other settings, COVID-19 has led to many public health problems in Iran, while informal sector employees have been suffering the most, particularly PLWANCDs, whose reduced income exacerbated their vulnerability in seeking required care (8). Worse still, the underlying preexisting conditions, ie, the high unemployment rate, insufficient unemployment insurance, and unpleasant economic conditions due to unilateral political and financial sanctions (9) against Iran, have led PLWANCDs to witness severe disruptions in their essential services. Ever since the COVID-19 outbreak initiated in late February 2020 in Iran, the Center for NCDs Management within the Ministry of Health \& Medical Education (MoHME) provided a set of measures to mitigate the adverse effects and manage the interaction of NCDs and COVID-19 (Table $1)$.

Table 1. NCDs-specific responses during COVID-19 in Iran

\begin{tabular}{ll}
\hline Domains & Responses \\
\hline Dietary & Appropriate diet for healthy people, PLWANCDs, and COVID-19 patients were outlined and realized \\
& through the media and service providers. All food service centers, including restaurants, were baned to serve \\
food inside to reduce the risk of infection by (observing the announced principles takeaway or delivery are & allowed), and a safe food shopping guide has been taught.
\end{tabular}

Physical activity

All sports gatherings were banned, low-risk ways of doing physical activity for the community, and PLWANCDs were taught continuously through the media.

Tobacco and alcohol and drug The hookah providers were closed, and drug and tobacco users were informed about the risk of more severe symptoms of COVID-19.

"Rumors (Alcohol consumption to prevent COVID-19) were denied, although unfortunately, some died.

Access to necessary healthcare services

Diagnosis and examination for COVID-19

Contact tracing and Contact prevention

Working conditions

Centers that did not admit COVID-19 cases were selected, to ensure secure access to services by PLWANCDs. Developed a national protocol to ensure receiving appropriate care and essential medicine during the pandemic. Community health workers based at the primary healthcare (PHC) centers facilities were instructed to make regular phone calls to train PLWANCDs for prevention and control of COVID-19 and encourage them to adhere to appropriate treatment and self-care. Almost 9 million people with pre-existing chronic medical conditions have received nationally recommended services so far.

In the National Protocol for the Diagnosis and Treatment of COVID-19; People with non-communicable diseases were considered susceptible and were given priority for active follow-up, even if they did not have symptoms.

After diagnosing each case of COVID-19, her/his contact was tracked, and the training was given to people in contact, especially for PLWANCDs.

PLWANCDs went on holiday or quarantine before others, and they are scheduled to return to work after all. * At the beginning of the epidemic, it was rumored on the social media that alcohol consumption is a preventive measure; Due to the illegal sale of alcohol and the existence of non-standard products (Methanol), some people lost their lives or were severely harmed. 
UHC strengthening for a balanced response to prevent and control NCDs in COVID-19 era

The rapidly unfolding story of the COVID-19 pandemic in many countries has diverted attentions to the potential upcoming pandemics as the biggest challenge to all societies. The extent and fundamental impact of the current crisis might lead many governments and health systems as well as the global agencies, i.e., UN, WHO, and others, to allocate and spend much of the resources to strengthen health systems and communities on emergency preparedness, monitoring, and surveillance of public health threat, ie, bacterial and viral infections (10). In a global economy, which is badly hit by the virus and is likely to face dramatic COVID-19 aftermath, advocating for greater resources to reduce NCDs in competition with various infectious threats might look utopia. NCDs, along with universal health coverage (UHC) and the unfinished agenda of emerging and reemerging diseases have been advocated as the main components of SDG3 during the last decade. However, keeping the balance of required investment on NCDs during the COVID-19 pandemic might become unconvincing for many governments that had just begun to climb the ladder of scaling up political support and technical capacity to tackle NCDs and their risk factors, among many challenges in their health system.

\section{What to do?}

NCDs and related risk factors will remain the greatest challenge to human development in the future. Although the current pandemic will be a fundamental game-changer for prioritization and resource allocation in many countries in years to come, we have ample evidence to conclude that NCDs will remain the main killer of people and is the costliest barrier to sustainable societies (11).

The UN, WHO, and the global governance of the health system should not let the current crisis fade recent global attempts in scaling up prevention and control of NCDs within national health systems and other relevant sectors. In this regard, strengthening UHC is fundamental to respond to the COVID-19 crisis. UHC provides a feasible platform for integration of NCDs services into primary health care (PHC) network, particularly in the LMICs.

We call upon all health activists and sustainable development advocates to proactively prevent the likely derail of the already retard governance of NCDs in many LMICs to reach sustainable development agenda by 2030 . In particular, health systems need to advocate for secure sustainable financial and other resources to overcome the challenge towards attaining SDG 3.8 and 3.4. The allocation of financial and nonfinancial resources for COVID-19 and NCDs and other diseases should be done through the lenses of $\mathrm{UHC}$ to maintain a balance between diseases to make the cost-effective use of resources.

Effective mechanisms should be fostered for meaningful multisectoral collaboration that is the key to reduce most risk factors of NCDs. Intersectoral collaboration is also the key to increase sin taxes on harmful products, eg, alcohol, tobacco, sugar, salt, and fat, as well as high-polluting vehicles, and spend the acquired extra resources on prevention and control of NCDs. In particular, the funds need to be directed towards innovative technologies for early detection, control of behavioral and metabolic risk factors, and provision of inexpensive and effective medicines for NCDs prevention and treatment. The use of mobile health is crucial in this regard, particularly within the LMICs. In addition, we encourage national and local governments to engage with the private and nonstate actors, ie, nongovernmental organizations (NGOs) and civil society through tax incentives and long-term/low-interest loans to invest on cost-effective and affordable technologies, medicines, and medical equipment for health promotion/ prevention and cure for main NCDs and relevant risk factors. Financial protection of citizens is essential in NCDs' care, particularly for the poor and vulnerable citizens. As countries have begun to provide financial packages to compensate for COVID-19 consequences, part of this package could be spent on insurance firms to pay towards NCDs' spiraling costs. WHO's best buys and Package of Essential NCDs Interventions (PEN) are cost-effective and available measures to be adopted in these tough circumstances to manage NCDs anywhere, including the LMICs. Moreover, functioning electronic health record (EHR) systems are crucial for necessary follow-ups, which is also a great asset for patients' identification and management during the pandemic.

\section{Conclusion}

COVID-19 taught us the need for fundamental governance reform to accommodate threats to the health system and the whole economy. Even after the current pandemic will be over, biological threats, natural or manmade, will still remain the Damocles Sword of sustainable development in all settings. It seems that as the world has become a global village, health systems are not equipped enough to deal with isolated communities from other parts of the world. Therefore, the direct and indirect spread of any outbreak to other parts of the globe is inevitable. The globalization phenomenon renders greater interaction between communicable and noncommunicable diseases in a different and more profound ways already observed during the last few months of the COVID-19 pandemic. Therefore, health systems need to become resilient enough to ensure the appropriate, effective, and meaningful response to the ongoing needs, ie, health promotion, prevention, early diagnosis and detection, treatment and rehabilitation of NCDs and related risk factors during and after the current COVID-19 crisis. In line with the principles of UHC, we must act now, more than ever, to rebuild our world in a way to harmonize efforts to live with pandemics and make our health systems resilient, balanced, and comprehensive enough to accommodate all threats to humanity, including both communicable and noncommuincable diseases.

\section{Acknowledgment}

We are grateful to anonymous reviewers and journal editors for their comments and suggestions. 
Conflict of Interests

AT and AO are members of the INCDC at the MoHMEIran. AO is Director-General for NCDs at the MoHME. We have no conflict of interest to disclose.

\section{References}

1.Legido-Quigley H, Asgari N, Teo YY, Leung GM, Oshitani H, Fukuda K, et al. Are high-performing health systems resilient against the COVID-19 epidemic? Lancet. 2020;395(10227):848-50.

2.WHO. Coronavirus disease 2019 (COVID-19) Situation Report $-83.2020$.

3. Raoofi A, Takian A, Akbari Sari A, Olyaeemanesh A, Haghighi H, Aarabi M. COVID-19 Pandemic and Comparative Health Policy Learning in Iran. Arch Iran Med. 2020;23(4):220-34.

4.Zheng YY, Ma YT, Zhang JY, Xie X. COVID-19 and the cardiovascular system. Nat Rev Cardiol. 2020.

5. Wu Z, McGoogan JM. Characteristics of and Important Lessons From the Coronavirus Disease 2019 (COVID-19) Outbreak in China: Summary of a Report of 72314 Cases From the Chinese Center for Disease Control and Prevention. JAMA. 2020.

6.Bakhtiari A, Takian A, Majdzadeh R, Haghdoost AA. Assessment and prioritization of the WHO "best buys" and other recommended interventions for the prevention and control of non-communicable diseases in Iran. BMC Public Health. 2020;20(1):333.

7.Nugent R, Bertram MY, Jan S, Niessen LW, Sassi F, Jamison $\mathrm{DT}$, et al. Investing in non-communicable disease prevention and management to advance the Sustainable Development Goals. Lancet. 2018;391(10134):2029-35.

8.Takian, A., Kiani, M.M. \& Khanjankhani, K. COVID-19 and the need to prioritize health equity and social determinants of health. Int J Public Health (2020). https://doi.org/10.1007/s00038-020-01398-z.

9. Takian A, Raoofi A, Kazempour-Ardebili S. COVID-19 battle during the toughest sanctions against Iran. Lancet. 2020;395(10229):1035-6.

10. World-Bank. World Bank Group Launches First Operations for COVID-19 (Coronavirus) Emergency Health Support, Strengthening Developing Country Responses 2020 [Available from:https://www.worldbank.org/en/news/press-release/2020/ 04/02/world-bank-group-launches-first-operations-for-covid19-coronavirus-emergency-health-support-strengtheningdeveloping-country-responses.

11. Smith PC, Anell A, Busse R, Crivelli L, Healy J, Lindahl AK, et al. Leadership and governance in seven developed health systems. Health Policy. 2012;106(1):37-49. 